\title{
BAO Plate Archive Project: Recent Results
}

\author{
G. A. Mikayelyan, G. M. Paronyan, N. M. Azatyan, G. R. Kostandyan, A. L. Samsonyan, H. \\ R. Andreasyan, H. V. Abrahamyan, D. H. Andreasyan, A. G. Suqiasyan, and
}

A. M. Mickaelian

NAS RA V. Ambartsumian Byurakan Astrophysical Observatory (BAO)

\begin{abstract}
We present the recent results of the Byurakan Astrophysical Observatory (BAO) Plate Archive Project that is aimed at digitization, extraction and analysis of archival data and building an electronic database and interactive sky map. BAO Plate Archive consists of some 37,000 photographic plates and films, obtained with $2.6 \mathrm{~m}$ telescope, $1 \mathrm{~m}$ and $0.5 \mathrm{~m}$ Schmidt telescopes and other smaller ones during 1947-1991 and then by digital methods since 1996. Its most important part, the famous Markarian Survey (or the First Byurakan Survey, FBS) 1874 plates were digitized in 2002-2007 and the Digitized FBS (DFBS, www. aras.am/Dfbs/dfbs.html) was created. New science projects have been conducted based on this low-dispersion spectroscopic material. Several other smaller digitization projects have been carried out as well, such as part of the Second Byurakan Survey (SBS) plates, photographic chain plates in Coma, where the blazar ON 231 is located and $2.6 \mathrm{~m}$ film spectra of FBS Blue Stellar Objects. However, most of the plates and films were not digitized. In 2015, we have started a project on the whole BAO Plate Archive digitization, creation of electronic database and its scientific usage. Armenian Virtual Observatory (ArVO, www.aras.am/Arvo/arvo.htm) database will accommodate all new data. The project lasted 4 years in 2015-2018. Later on, the project was renovated for 2020-2021. The final result will be an Electronic Database and online Interactive Sky map to be used for further research projects.
\end{abstract}

Keywords: photographic plates - photographic films - digitization - astrometry - photometry - spectroscopy - astronomical archives - databases - virtual observatories

\section{Introduction}

The astronomical plate archives created on the basis of numerous observations at many observatories are the most important part of the astronomical observational heritage. The necessity of digitization of astronomical plates was emphasized and current progress in various national and international projects was given at Astroplate workshops (e. g. Hudec 2014, Kazantseva 2014, Nesci et al. 2014, Osborn 2014, Stupka \& Benesova 2014).

Byurakan Astrophysical Observatory (BAO) Plate Archive is one of the largest astronomical archives in the world and is considered to be BAO main observational treasure. It is the results of decades' hard work of Armenian astronomers and the work of BAO telescopes and other expensive equipment, as well as the results of their activities. Today BAO archive holds some 37,000 astronomical plates, films or other carriers of observational data. However, previous observational and informational registration methods currently do not make it available to wide range of scientists, and especially its usage for solution of new research problems. Digitization of BAO plates is a significant contribution to the Wide-Field Plate Data Base (WFPDB) developed in Sofia, Bulgaria (Tsvetkov \& Tsvetkova, 2012).

A project on Digitization of BAO Plate Archive and creation of BAO Interactive Astronomical Database (shortly BAO Plate Archive project, BAO PAP) has started in February 2015. It was aimed at preservation of BAO valuable observational material accumulated during 1947-1991, creation

*gormick@mail.ru 
of full Database of all BAO observations, creation of BAO Interactive Sky Map with visualization of all observations and quick access to the data, development and accomplishment of new research projects based on the existing observational material, and integration of $\mathrm{BAO}$ observations into the international databases. A number of BAO young astronomers were involved in this project and it lasted 4 years. Later on, the project was renovated for 2020-2021 to complete the full tasks. Project objectives are the preservation of BAO observational archive, preservation of scientific information contained in photographic plates and other careers, creation of opportunity of dissemination and wide usage of observational data, putting in correspondence of observational material to modern standards and usage methods, proposing new science projects and creation of possibility of their further accomplishment, and making BAO activities visible.

A short description of BAO Plate Archive was given by Mickaelian (2014) and more detailed papers are given in Mickaelian et al. (2016, 2017, 2020).

\section{BAO telescopes and observing programs}

BAO observers worked with a number of BAO telescopes during 1947-1991 and obtained several dozens of thousands of plates, films and other products. The table gives general understanding on observations of $10 \mathrm{BAO}$ telescopes that worked on photographic photometry, electrophotometry, slit and objective prism spectroscopy, and polarimetry of many thousands astronomical objects.

Table 1. Overview of BAO telescopes and produced observational material.

\begin{tabular}{|l|c|c|l|r|}
\hline Telescopes & Sizes $(\mathrm{cm})$ & Years & Observing methods & Plates \\
\hline 5" double-astrograph & $13 /$ & $1947-1950$ & photometry & 3000 \\
\hline 6" telescope & $15 /$ & $1947-1950$ & photometry & 3000 \\
\hline 8" Schmidt & $20 / 20 / 31$ & $1949-1968$ & photometry & 4500 \\
\hline 20" Cassegrain (AZT-14) & $51 / 400$ & $1955-1991$ & electropolarimetry & $\mathrm{n} / \mathrm{a}$ \\
\hline 10" telescope-spectrograph & $25 /$ & $1953-19 ? ?$ & spectroscopy & $\mathrm{n} / \mathrm{a}$ \\
\hline Nebular spectrograph (ASI-1) & $\mathrm{n} / \mathrm{a}$ & $1953-19 ? ?$ & spectroscopy & $\mathrm{n} / \mathrm{a}$ \\
\hline 16" Cassegrain & $41 / 800$ & $1952-1991$ & electrophotometry & $\mathrm{n} / \mathrm{a}$ \\
\hline 21" Schmidt (AZT-1) & $53 / 53 / 183$ & $1955-1991$ & photometry & 12000 \\
\hline 40" Schmidt (AZT-10) & $102 / 132 / 213$ & $1960-1991$ & photometry, spectroscopy & 7500 \\
\hline ZTA-2.6 & $264 / 1016$ & $1975-1991$ & photometry, spectroscopy & 7000 \\
\hline BAO all telescopes & & $\mathbf{1 9 4 7 - 1 9 9 1}$ & & $\mathbf{3 7 0 0 0}$ \\
\hline
\end{tabular}

We give in Table 1 an overview of BAO telescopes and produced observational material. Telescope "Sizes" are given for the mirror and focal length for classical telescopes and for the correcting lens, mirror and focal length for Schmidt type telescopes. Here we list the main observational projects accomplished on the three most important BAO telescopes $(2.6 \mathrm{~m}$ classical reflector, $1 \mathrm{~m}$ Schmidt and $0.5 \mathrm{~m}$ Schmidt; Figure 1).

\section{1" (0.5m) Schmidt telescope:}

- Polarization of cometary nebula NGC 2261

- Nuclei of nearby Sa and Sb galaxies

- Nuclei of nearby Sc galaxies

- Search for flare stars in Pleiades

- Search for flare stars in Orion

- Search for flare stars in NGC 7000 (Cygnus)

- Search for flare stars in Praesepe 


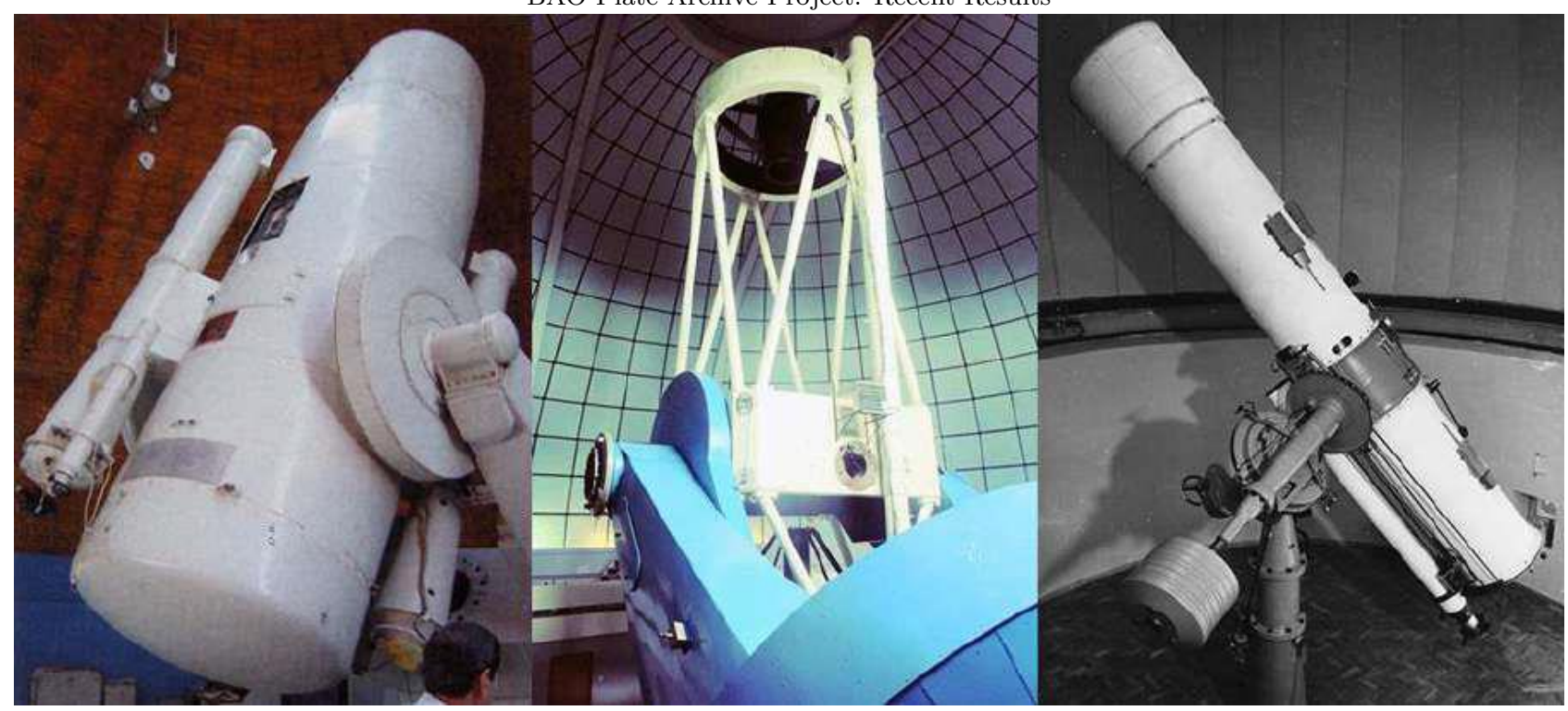

Figure 1. BAO most important telescopes (from left to right): 1m Schmidt, $2.6 \mathrm{~m}$ classical reflector and $0.5 \mathrm{~m}$ Schmidt.

- Search for flare stars in Taurus Dark Clouds (TDC)

- Variability of Markarian galaxies

- Monitoring of extragalactic supernovae in certain areas

\section{0" (1m) Schmidt telescope:}

- Detailed colorimetry of bright galaxies

- First Byurakan Survey (FBS, Markarian survey; Markarian et al. 1989)

- Search for flare stars in Pleiades

- Search for flare stars in Orion

- Search for flare stars in NGC 7000 (Cygnus)

- Search for flare stars in Praesepe

- Search for flare stars in Taurus Dark Clouds (TDC)

- Second Byurakan Survey (SBS; Stepanian 2005)

- Extension of the FBS in the Galactic Plane

\section{ZTA-2.6m telescope:}

- Morphological study of Markarian galaxies

- Investigation of star clusters

- Investigation of groups and clusters of galaxies

- Spectroscopy FBS blue stellar objects

- Spectroscopy FBS late-type stars

- Spectroscopy SBS galaxies and stellar objects (BAO/SAO) 
- Direct images of the central regions of Markarian galaxies

- Spectroscopy of $\mathrm{T}$ Tauri and flare stars

- Spectroscopy of Byurakan-IRAS Galaxies (BIG objects)

- Spectroscopy of ROSAT AGN candidates (BAO/HS/OHP/INAOE)

Summarizing, the main observational projects run on these telescopes were:

21" (0.5m) Schmidt: Polarization of cometary nebula NGC 2261, Nuclei of nearby Sa and Sb galaxies, Nuclei of nearby Sc galaxies, Search for are stars in Pleiades, Orion, NGC 7000 (Cygnus), Praesepe and Taurus Dark Clouds (TDC), Variability of Markarian galaxies, Monitoring of extragalactic supernovae in certain areas, etc.;

40" (1m) Schmidt: the First Byurakan Survey (FBS, Markarian survey; Markarian et al. 1989), the Second Byurakan Survey (SBS; Stepanian 2005), Extension of the FBS in the Galactic Plane, Detailed colorimetry of bright galaxies, Search for are stars in Pleiades, Orion, NGC 7000 (Cygnus), Praesepe and Taurus Dark Clouds (TDC), etc.;

ZTA-2.6m telescope: Morphological study of Markarian galaxies, Investigation of star clusters, Investigation of groups and clusters of galaxies, Spectroscopy of FBS blue stellar objects, FBS latetype stars, SBS galaxies and stellar objects (BAO/SAO), T Tauri and are stars, Byurakan-IRAS Galaxies (BIG objects) and ROSAT AGN candidates (BAO/HS/OHP/INAOE), and Direct images of the central regions of Markarian galaxies.

Especially efficient were Byurakan spectroscopic surveys accomplished by Markarian and colleagues with $1 \mathrm{~m}$ Schmidt telescope: FBS and SBS.

\section{BAO Plate Archive Project}

The digitization of astronomical plates and films pursues not only the maintenance task, but also it will serve as a source for new scientific research and discoveries, if only the digitized material runs according to modern standards and, due to its accessibility, it will become an active archive. The project is aimed at compilation, accounting, digitization of BAO observational archive photographic plates and films, as well as their incorporation in databases with modern standards and methods, providing access for all observational material and development of new scientific programs based on this material.

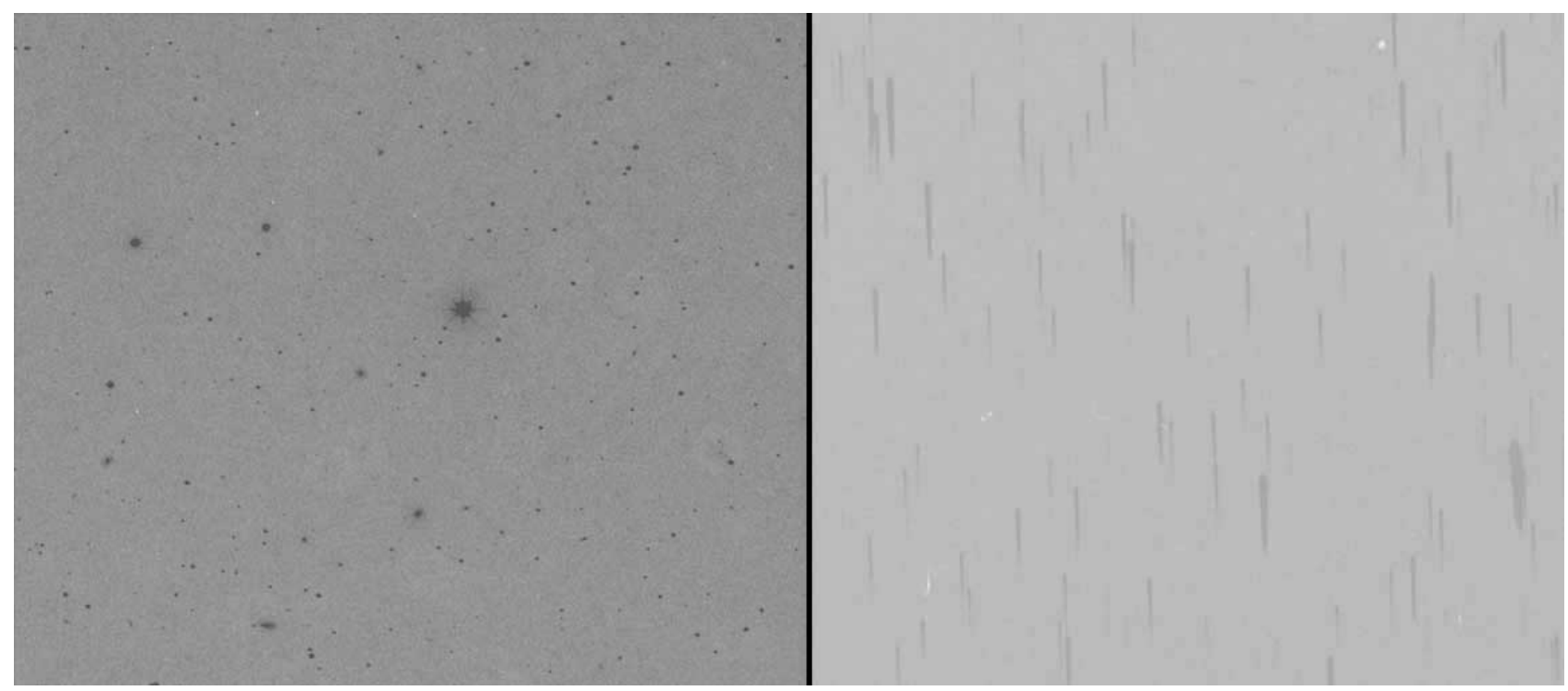

Figure 2. Example of photometric and spectroscopic plates.

Scientific Programs Board (SPB) was created to evaluate the existing observational material, to select sets of priorities to be scanned first and to propose new research projects. It consists of 


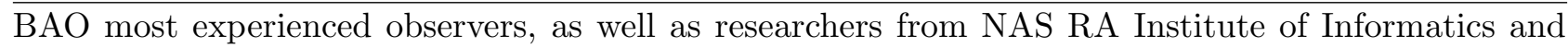
Automation Problems (IIAP) are involved for their experience in computer science related to databases and computational methods.

Project Executing Team (PET) consists of more than 10 members led by the Head of BAO Astroinformatics Department Gor Mikayelyan, and the members are involved in scanning and reduction of data.

The project has started in February 2015 and it lasted 4 years. In August 2020 the project was renovated and now the digitization of the plates is finished. Altogether 30121 photographic plates and films were digitized. About half of them are photometric plates of different sizes and the other ones are spectroscopic plates, photometric chains, spectroscopic chains, etc. In Figures 2, 3 and 4 we give the examples of digitized plates.

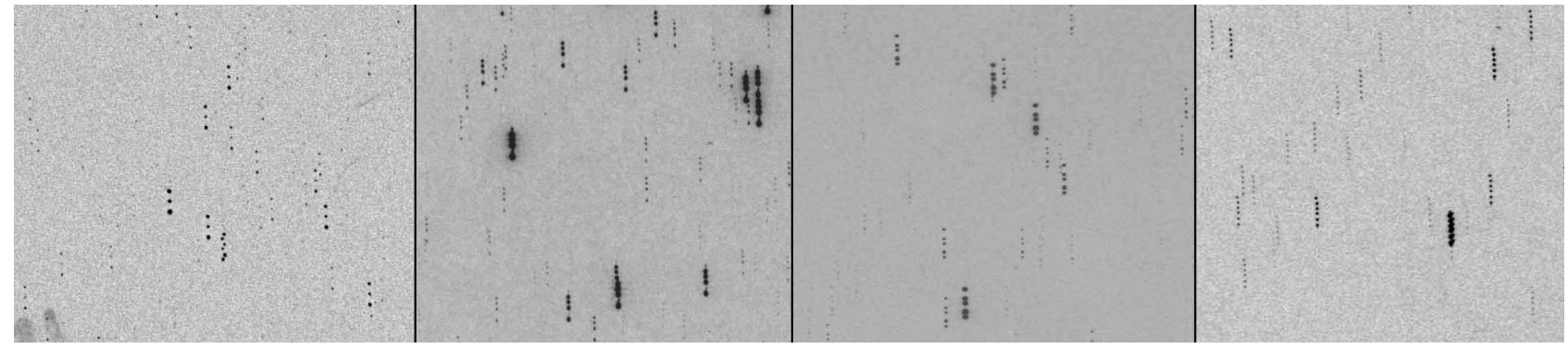

Figure 3. Example of photometric chains.

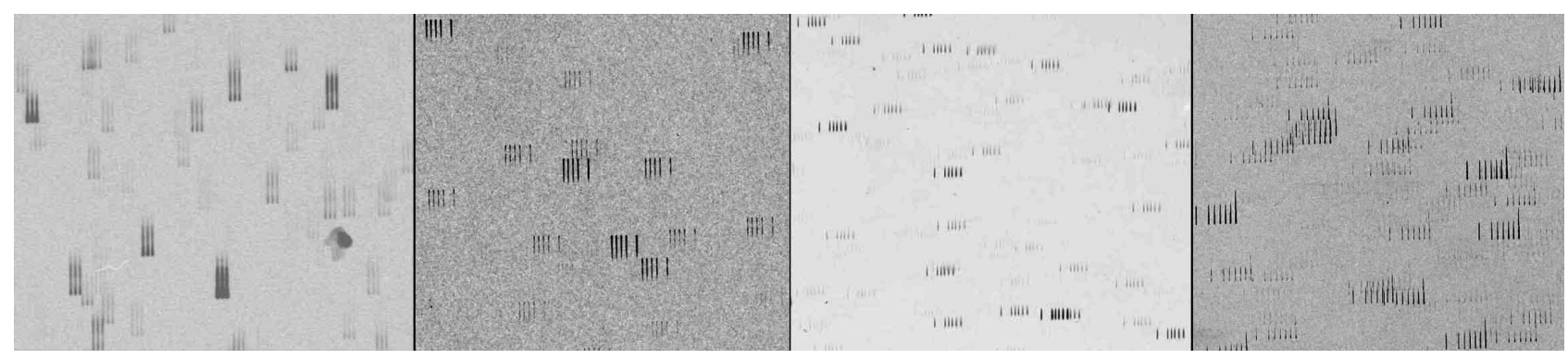

Figure 4. Example of spectroscopic chains.

In May 2021 we have started the works of astrometric calibration and now about 5000 plates already have an astrometric solution. We use https://astrometry.net webpage for astrometric calibration of the plates. We plan to finish the astrometric calibration of all plates in December 2021.

\section{Digitized First Byurakan Survey}

A number of digitization projects have been accomplished at $\mathrm{BAO}$, including the most important one, Digitized First Byurakan Survey (DFBS; http://www. aras . am/Dfbs/dfbs .html; Massaro et al. 2008, Mickaelian et al. 2007) based on the digitization of the famous Markarian Survey (Markarian et al., 1989). Its main scanning and resulting features are given in Table 2.

We give in Figure 5 (left panel) a piece of visualized DFBS field together with its corresponding DSS2 area. Some 40,000,000 DFBS low-dispersion spectra have been extracted from 1874 plates, measured and analyzed by means of the dedicated software bSpec (Figure 5, right panel), written by Giuseppe Cirimele. The spectra extraction and analysis software is described in Mickaelian et al. (2010) and Knyazyan et al. (2011). DFBS plate database is available in Vizier, Strasbourg (Mickaelian et al., 2005). 
Table 2. Main scanning and resulting characteristics of the DFBS

\begin{tabular}{|l|l|}
\hline Items & Description \\
\hline Teams & Byurakan Astrophys. Obs., Univ. Roma "La Sapienza", Cornell Univ. \\
\hline Years & $2002-2005$ \\
\hline Instrument & Epson Expression 1680 Pro scanner \\
\hline Scanning options & $1600 \mathrm{dpi}(15.875 \mu$ pix size $), 16$ bit, transparency mode, "scanfits" \\
\hline Plate size & $9601 \times 9601$ pix, $176 \mathrm{MB}$ file \\
\hline Spectra & $107 \times 5$ pix $(1700 \mu$ in length $)$ \\
\hline Dispersion & $33 \AA /$ pix average $(22-60 \AA /$ pix $), 28.5$ at $\mathrm{H}_{\gamma}$ \\
\hline Spectral resolution & $50 \AA$ (average $)$ \\
\hline Astrometric solution & $1 "$ rms accuracy \\
\hline Scale & $1.542^{\prime \prime} /$ pix \\
\hline Photometry & $0.3^{m}$ accuracy \\
\hline Data volume & 1874 plates, $\sim 400 \mathrm{~GB}$ \\
\hline Number of objects & $\sim 20,000,000(\sim 40,000,000$ spectra $)$ \\
\hline
\end{tabular}
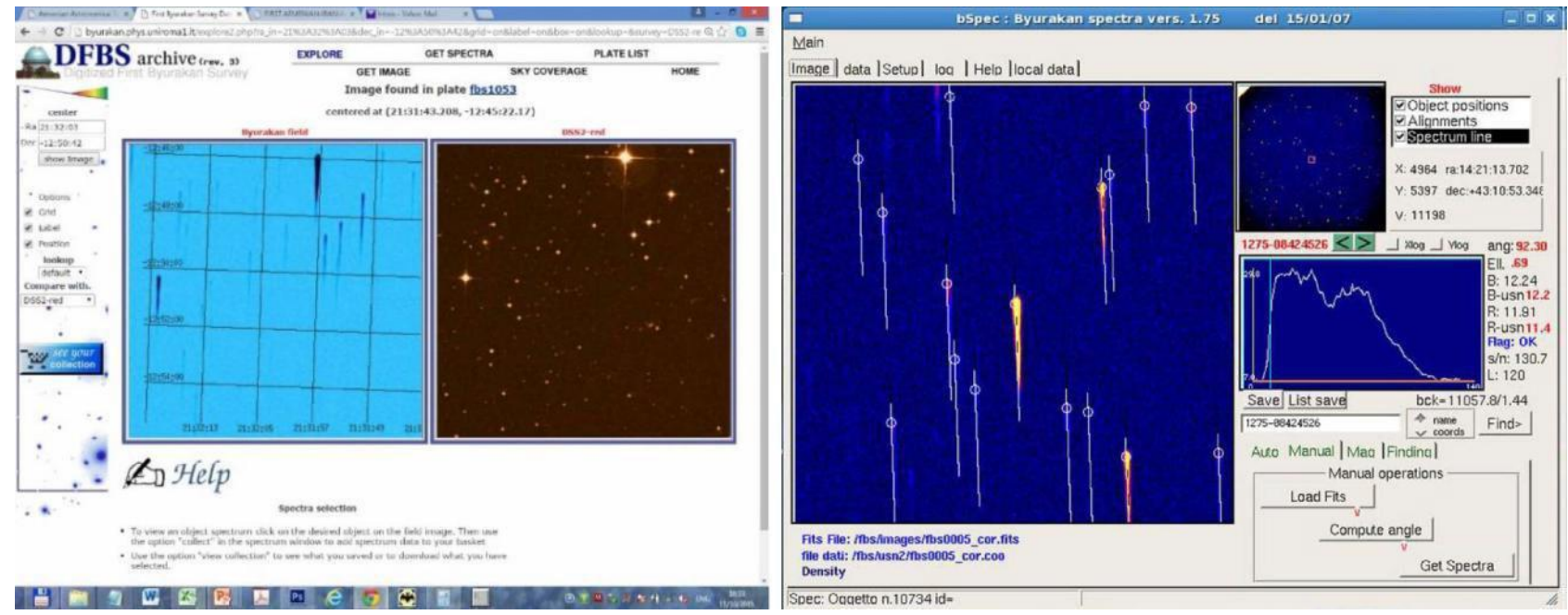

Figure 5. Left: DFBS data visualization with comparison of similar DSS2 field. Right: DFBS spectra extraction and analysis software bSpec.

\section{Summary}

BAO Plate Archive is one of the most important astronomical databases. At present the main part of the project has been accomplished, the scanning of the plates has produced more than 30,000 digital images of roughly $200 \mathrm{MB}$ files (each image). All they have been stored and double copies are available.

BAO Plate Archive Project will lead to preservation of BAO valuable observational material obtained during 1947-1991. However, our goal is not only to create a passive archive of scanned plates and films, but also to make use of especially those fields, where more studies are possible. Proper motion and variability studies are most important, as time domain material is contained in historical plates. Such possibilities based on DFBS were shown by Mickaelian et al. (2006a); DFBS as a unique database for proper motion, variability studies, and object classification. New variable stars discovered on digitized plates of Moscow collection was reported by Sokolovsky et al. (2014).

There are a number of further possible research projects that will be conducted having the plates digitized:

- Correction of ephemerides of known asteroids and search for new asteroids (ex. Berthier et al. 2009, Mickaelian et al. 2019, Thuillot et al. 2007)

- Discovery and study of variable stars (ex. Mickaelian et al. 2011, Nesci et al. 2009) 
- Revealing high proper motion stars (ex. Mickaelian \& Sinamyan 2010)

- Study of variability of known blazars and discovery of new blazars

- Revealing Novae and Supernovae progenitors (Nesci et al. 2009)

- Discovery of new QSOs

- Discovery of new white dwarfs (ex. Sinamyan \& Mickaelian 2011)

- Discovery of new late-type stars (ex. Gigoyan et al. 2010, 2019)

- Discovery of optical sources of gamma-ray bursts

- Optical identifications of X-ray, IR and radio sources (ex. Hovhannisyan et al. 2009, Mickaelian \& Gigoyan 2006, Mickaelian \& Sargsyan 2004, Mickaelian et al. 2006b).

\section{Acknowledgements}

BAO Plate Archive Project team would like to thank BAO Administration for supporting the project. We also would like to thank the astrometry.net team because the astrometric calibration of the plates became possible thanks to https://astrometry . net webpage.

\section{References}

Berthier J., Sarkissian A., Mickaelian A. M., Thuillot W., 2009, "European Planetary Science Congress 2009, held 14-18 September in Potsdam, Germany, p. 526

Gigoyan K. S., Sinamyan P. K., Engels D., Mickaelian A. M., 2010, Astrophysics, 53, 123

Gigoyan K. S., Kostandyan G. R., Gigoyan K. K., Zamkotsian F., Sarkissian A., 2019, Astrophysics, 62 , 573

Hovhannisyan L. R., Mickaelian A. M., Weedman D., et al. 2009, AJ, 251, 138

Hudec R., 2014, in Proc. Astroplate-2014 Conf., Eds. L. Mišková \& S. Vítek. Prague, p. 1

Kazantseva L., 2014, in Proc. Astroplate-2014 Conf., Eds. L. Mišková \& S. Vítek. Prague, p. 13

Knyazyan A., Mickaelian A. M., Astsatryan H., 2011, Int. J. "Inform. Theories and Appl.", 18,243

Markarian B. E., Lipovetsky V. A., Stepanian J. A., et al. 1989, Comm. SAO, 62, 5

Massaro E., Mickaelian A. M., Nesci R., Weedman D., (Eds.) 2008, The Digitized First Byurakan Survey, ARACNE Editrice, Rome, 78p.

Mickaelian A. M., 2014, in Proc. Astroplate-2014 Conf., Eds. L. Mišková \& S. Vítek. Prague, p. 109

Mickaelian A. M., Gigoyan K. S., 2006, A\&A, 455, 765

Mickaelian A. M., Sargsyan L. A., 2004, Astrophysics, 47, 213

Mickaelian A. M., Sinamyan P. K., 2010, MNRAS, 407, 681

Mickaelian A. M., Hagen H.-J., Sargsyan L. A., Mikayelyan G. A., 2005, Catalog No. VI/116 at CDS, Strasbourg

Mickaelian A. M., Gigoyan K. S., Nesci R., Rossi C., 2006a, Mem.S.A.It., 77, 1159

Mickaelian A. M., Hovhannisyan L. R., Engels D., et al. 2006b, A\&A, 449, 425

Mickaelian A. M., Nesci R., Rossi C., et al. 2007, A\&A, 464, 1177

Mickaelian A. M., Sargsyan L. A., Nesci R., et al. 2010, ASP Conf. Series, Vol. 434: Astronomical Data Analysis Software and Systems XIX, p. 325

Mickaelian A. M., Mikayelyan G. A., Sinamyan P. K., 2011, MNRAS, 415, 1061

Mickaelian A. M., Abrahamyan H. V., Andreasyan H. R., et al. 2016, in Astronomical Surveys and Big Data. Eds. A. M. Mickaelian, A. Lawrence \& T. Yu. Magakian. ASP Conf. Ser., V. 505, p. 262

Mickaelian A. M., Gigoyan K. S., Gyulzadyan M. V., et al. 2017, ComBAO, 64, 102

Mickaelian A. M., Sarkissian A., Berthier J., Meftah M., Thuillot W., Vachier F., 2019, Icarus, 330, 5 
Mickaelian A. M., Gigoyan K. S., Mikayelyan G. A., et al. 2020, ComBAO, 67 2, 293

Nesci R., Mickaelian A. M., Rossi C., 2009, ATel, 2338, 1

Nesci R., Bagaglia M., Nucciarelli G., 2014, in Proc. Astroplate-2014 Conf., Eds. L. Mišková \& S. Vítek. Prague, p. 75

Osborn W., 2014, in Proc. Astroplate-2014 Conf., Eds. L. Mišková \& S. Vítek. Prague, p. 15

Sinamyan P. K., Mickaelian A. M., 2011, Astrophysics, 54, 403

Sokolovsky K. V., Antipin S. V., Zubareva A. M., et al. 2014, ARep, 58, 319

Stepanian J. A., 2005, RMxAA, 41, 155

Stupka J., Benesova E., 2014, in Proc. Astroplate-2014 Conf., Eds. L. Mišková \& S. Vítek. Prague, p. 31

Thuillot W., Berthier J., Sarkissian A., et al. 2007, Highlights of Astronomy, 14, 616

Tsvetkov M., Tsvetkova K., 2012, in Proc. IAU S285: New Horizons in Time-Domain Astronomy. Cambridge Univ. Press, p. 417 